\title{
Improved virtual surgical planning with 3D- multimodality image for malignant giant pelvic tumors
}

This article was published in the following Dove Press journal: Cancer Management and Research

\section{Xiang Fang ${ }^{1, *}$ \\ Zeping Yu ${ }^{1, *}$ \\ Yan Xiong' \\ Fang Yuan ${ }^{2}$ \\ Hongyuan $\mathrm{Liu}^{3}$ \\ Fan $\mathrm{Wu}^{4}$ \\ Wenli Zhang' \\ Yi Luo' \\ Liuhong Song 5 \\ Chongqi Tu' \\ Hong Duan'}

'Department of Orthopedics, West China School of Medicine/West China Hospital, Sichuan University, Chengdu, Sichuan, People's Republic of China;

${ }^{2}$ Department of Radiology, West China School of Medicine/West China Hospital, Sichuan University, Chengdu, Sichuan, People's Republic of China; ${ }^{3}$ Department of Orthopedics, Sichuan Provincial Fifth People's Hospital, Chengdu, Sichuan, People's Republic of China; ${ }^{4}$ Department of Orthopedics, Fourth People's Hospital of ZiGong, Sichuan, People's Republic of China; ${ }^{5}$ Department of Orthopedics, People's Hospital of Pengzhou, Sichuan, People's Republic of China

*These authors contributed equally to this work

Correspondence: Hong Duan Department of Orthopedics, West China School of Medicine/West China Hospital, Sichuan University, 37 Guo Xue Lane, Chengdu, Sichuan 610064, People's Republic of China

Tel/fax +86 2885422578

Email duanhong1970@I26.com
Purpose: We sought to assess the early clinical outcome of 3D-multimodality image (3DMMI)based virtual surgical planning for resection and reconstruction of malignant giant pelvic tumors. Patients and methods: In this retrospective case-control study, surgery was planned and performed with 3DMMI-based patient-specific instruments (PSI) in 13 patients with giant pelvic malignancy and without 3DMMI-based PSI in the other 13 patients. In the 3DMMI group, 3DMMI was utilized, taking advantages of computed tomography (CT), contrast-enhanced CT angiography (CTA), contrast-enhanced magnetic resonance imaging (MRI), contrast-enhanced magnetic resonance neurography (MRN), which could reveal the whole tumor and all adjacent vital structures. Based on these 3DMMI, virtual surgical planning was conducted and the corresponding PSI was then designed. The median follow-up was 8 (3-24) months. The median age at operation was 37.5 (17-64) years. The mean tumor size in maximum diameter was 13.3 $\mathrm{cm}$. Surgical margins, intraoperative and postoperative complications, duration of surgery, and intra-operative blood loss were analyzed.

Results: In the non-3DMMI group, the margins were wide in six patients (6/13), marginal in four (4/13), wide-contaminated in two (2/13), and intralesional in one (1/13). In the 3DMMI group, the margins were wide in 10 patients $(10 / 13)$, marginal in three $(3 / 13)$, and there were no wide-contaminated or intralesional margins. The 3DMMI group achieved shorter duration of surgery $(P=0.354)$ and lower intraoperative blood loss $(P=0.044)$ than the non-3DMMI group. Conclusion: The 3DMMI-based technique is advantageous to obtain negative surgical margin and decrease surgical complications related to critical structures injury for malignant giant pelvic tumor.

Keywords: surgical planning, 3D-multimodality image, pelvic tumor, patient-specific instruments, surgical margin

\section{Introduction}

The surgical treatment of malignant pelvic bone tumors is challenging, due to the complex anatomy of the pelvis itself and the large size of the tumor. Before the 1980s, hemipelvic amputation was the main treatment for pelvic malignancies. ${ }^{1}$ Since 1978, Steel reported limb salvage procedure with internal pelvic resection and femoral head $(\mathrm{FH})$ exclusion for the first time, and with the advances in imaging, surgery, anesthesiology, and prosthesis, limb salvage therapy was gradually being applied to the treatment of malignant pelvic bone tumors. ${ }^{1}$ However, the high rate of recurrence and complications has remained troubling. ${ }^{2-5}$ Apart from the surgeons' technical level, a more important reason lies in the fact that surgeons need to build the tumor and its 
adjacent structures in the brain with each separate image, such as roentgenogram, computed tomography (CT) scan, and magnetic resonance imaging (MRI), which may result in inaccurate resection and reconstruction. Since the 1990s, with the rapid advancement of computer technology and the in-depth study of image registration algorithms, image fusion technology, especially 3D-multimodality image (3DMMI) has begun to enter the field of clinical practice. It makes full use of the advantages of various imaging technologies and presents $3 \mathrm{D}$ images objectively in the computer, instead of the brain. At present, 3DMMI has been applied in the field of neurosurgery, but no relevant reports have been found in bone tumors. ${ }^{6}$ The aim of this study is to assess the clinical significance and early outcome of 3DMMI-based virtual surgical planning for resection and reconstruction of malignant giant pelvic tumors.

\section{Patients and methods}

This is a retrospective case-control study (Levels of Evidence: III). The inclusion criteria were 1) malignant pelvic bone tumors; 2) giant soft tissue mass; 3) maximum diameter of the tumor $>10 \mathrm{~cm}$; and 4) planned for limb salvage surgery. A total of 26 patients from January 2015 to June 2018 were identified in our center. The gender ratio was $1: 1$. The median age at operation was 37.5 (17-64) years. Biopsy was done before surgery, and the pathological findings included 10 osteosarcoma, seven chondrosarcoma, four metastatic bone disease, three malignant giant cell bone tumors, one rhabdomyosarcoma, and one plasmacytoma. One patient $(1 / 26)$ had type I resection; one (1/26) had type I+II; four $(4 / 26)$ had type I+IV; three (3/26) had type I+II+IV; nine (9/26) had type II; and eight (8/26) had type II+III. ${ }^{7}$

Radiographic assessment included roentgenograph, CT, contrast-enhanced CT angiography (CTA), contrast-enhanced MRI, contrast-enhanced magnetic resonance neurography (MRN), and whole body bone scan. Thirteen patients underwent surgery with 3DMMI-based virtual surgical planning and 3D-printed patient-specific instruments (PSI), and they were identified as the 3DMMI group. The other 13 patients underwent conventional surgery with surgical planning based on each separate image without PSI, and they were identified as non-3DMMI group. Detailed information regarding each group is shown in Table 1.

In the 3DMMI group, 3DMMI was conducted through affine and diffeomorphic registration algorithm in open source software Advanced Normalization Tools (ANTs) before biopsy or upon completion of neoadjuvant chemotherapy. Then, the tumor and all adjacent structures
Table I Patients' characteristics

\begin{tabular}{|c|c|c|c|}
\hline & $\begin{array}{l}\text { Non-3DMMI } \\
\text { group }\end{array}$ & $\begin{array}{l}\text { 3DMMI } \\
\text { group }\end{array}$ & $P$-value \\
\hline Age (years) & $39.8 \pm 16.2$ & $37.9 \pm 14.9$ & 0.746 \\
\hline Male & $7(53.8)$ & $6(46.2)$ & \\
\hline Histological type & & & 0.707 \\
\hline Osteosarcoma & 4 & 6 & \\
\hline Chondrosarcoma & 4 & 3 & \\
\hline Metastatic & 3 & 1 & \\
\hline Malignant GCT & 1 & 2 & \\
\hline Plasmacytoma & 0 & 1 & \\
\hline Rhabdomyosarcoma & I & 0 & \\
\hline Tumor size $(\mathrm{cm})^{\mathrm{a}}$ & $13.1 \pm 2.08$ & $13.5 \pm 1.66$ & 0.681 \\
\hline Type of surgical resection & & & 0.709 \\
\hline I & 0 & 1 & \\
\hline $\mathrm{I}+\mathrm{II}$ & 1 & 0 & \\
\hline $\mathrm{I}+\mathrm{IV}$ & 2 & 2 & \\
\hline $\mathrm{I}+\mathrm{II}+\mathrm{IV}$ & 1 & 2 & \\
\hline II & 6 & 3 & \\
\hline II+III & 3 & 5 & \\
\hline
\end{tabular}

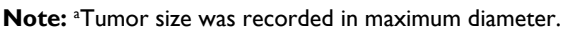
Abbreviation: 3DMMI, 3D-multimodality image; GCT, giant cell tumor of bone.

in the pelvis were segmented by using a level set-based segmentation algorithm implemented in open source software ITK-snap. ${ }^{8}$ After quality assurance and manual correction (accuracy of registration $>95 \%$, maximum segmentation error $<2 \mathrm{~mm}$ compared with the raw DICOM data), the finished 3DMMI was loaded in Creo 2.0 (PTC, Needham, MA, USA) to perform virtual surgical planning. The whole tumor (including both the bone and the soft tissue components) and its adjacent edema areas, vital nerves, vessels, and organs were identified and evaluated in both 3D and sliced 2D level to avoid the unnecessary injuries and to improve the understanding of the whole surgery (Figure 1). The resection plane was determined by the extent of tumor edema in 3DMMI and the need for functional reconstruction of the pelvis. Then, the corresponding PSI were designed and fabricated by 3D-printing technology to maximize the replication of the plan intraoperatively. 3D-printed model of the pelvis was also manufactured to facilitate intraoperative visual and haptic perception. During surgery, soft tissues were carefully identified and dissected according to the 3DMMI in cellphone (Video S1). The app used for 3DMMI was 3D PDF Reader 3.4 (Tech Soft 3D, Bend, OR, USA). After the exposure of specific bones for cutting, PSI was positioned and fixed on the corresponding bone surface with K-wires. The PSI served as mechanical support for cutting tools to help achieve the desired resection planes (Figure 2). Then, the PSI and K-wires were removed. After the 


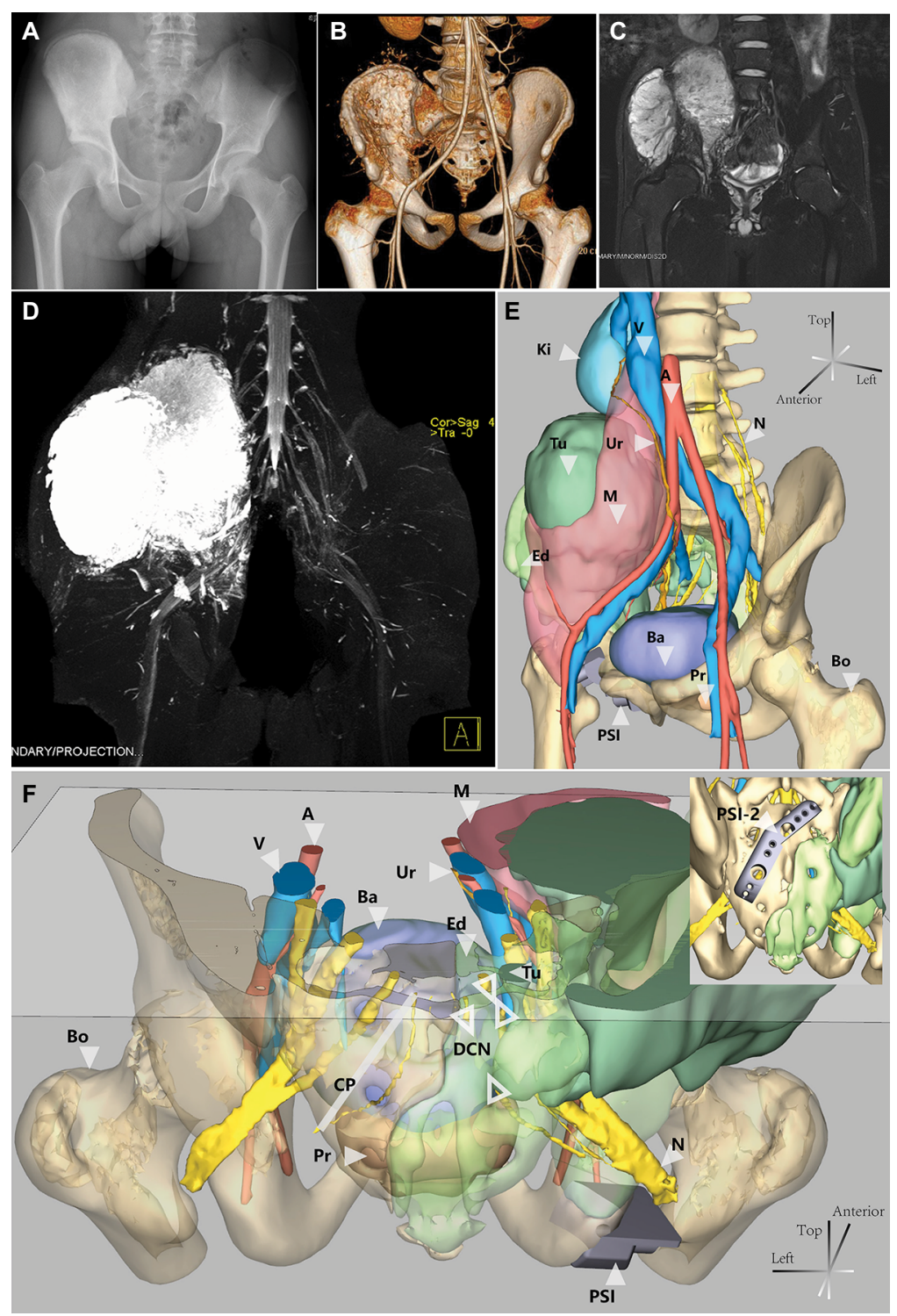

Figure I Each separate radiographic image and the 3DMMI of a 16-year-old boy with osteosarcoma with massive involvement.

Notes: (A) Plain film showing osteogenic lesion of the right ilium and periacetabulum. (B) 3D-CT images combined with contrast-enhanced CTA. (C) Contrast-enhanced MRI showing large soft tissue mass in the pelvis. (D) Contrast-enhanced MRN showing the sacral nerves and the tumor. (E) Anterolateral view of 3DMMI showing the tumor extended from periacetabulum to kidney level with involvement of the sacrum. Although pushed medially, the right external iliac vessels and ureter were free from the tumor due to the protection of the iliacus muscle and psoas major muscle. (F) Posterior and transverse plane of 3DMMI. Massive sacrum bone edema was noted. The right second and third sacral nerves $(\mathrm{DCN})$ were discontinued, while the left were intact, which might indicate involvement of the nerves. This information was very useful for accurate preoperative planning. PSI were also designed to help the resection, according to the virtual surgical planning.

Abbreviations: 3DMMI, 3D-multimodality image; A, artery; Ba, bladder; Bo, bone, translucent; CP, cutting plane; CTA, computed tomography angiography; DCN, discontinued nerves; Ed, edema, translucent, considered to be bone invasion; Ki, kidney; M, muscle, translucent; MRN, magnetic resonance neurography; MRI, magnetic resonance imaging; N, nerve; Pr, prostate; PSI, patient-specific instrument; Tu, tumor; Ur, ureter; $\mathrm{V}$, vein.

tumor resection, another pack of PSI was used for further reconstruction of the pelvis (Figure 3). During the whole process, the 3DMMI virtual plan and the 3D-printed model of the pelvis were repeatedly reviewed whenever necessary.

In the non-3DMMI group, the surgical plans were made based on each separate plain radiograph, CT and MRI. And these images were also repeatedly reviewed during surgery, whenever necessary. Surgical approach and closure for both groups were carried out in standard fashions. The method of reconstruction or whether reconstruction was performed following disruption of the pelvic ring was decided on a case by case basis. And we aimed in all patients of both groups to achieve wide surgical margins while maintaining vital anatomic structures and with consideration given to functional reconstruction of additional margin resection.

Margins and diagnosis were confirmed by the final specimen. Neo-adjuvant and adjuvant oncological therapy was determined by the histological diagnosis, the stage of the 

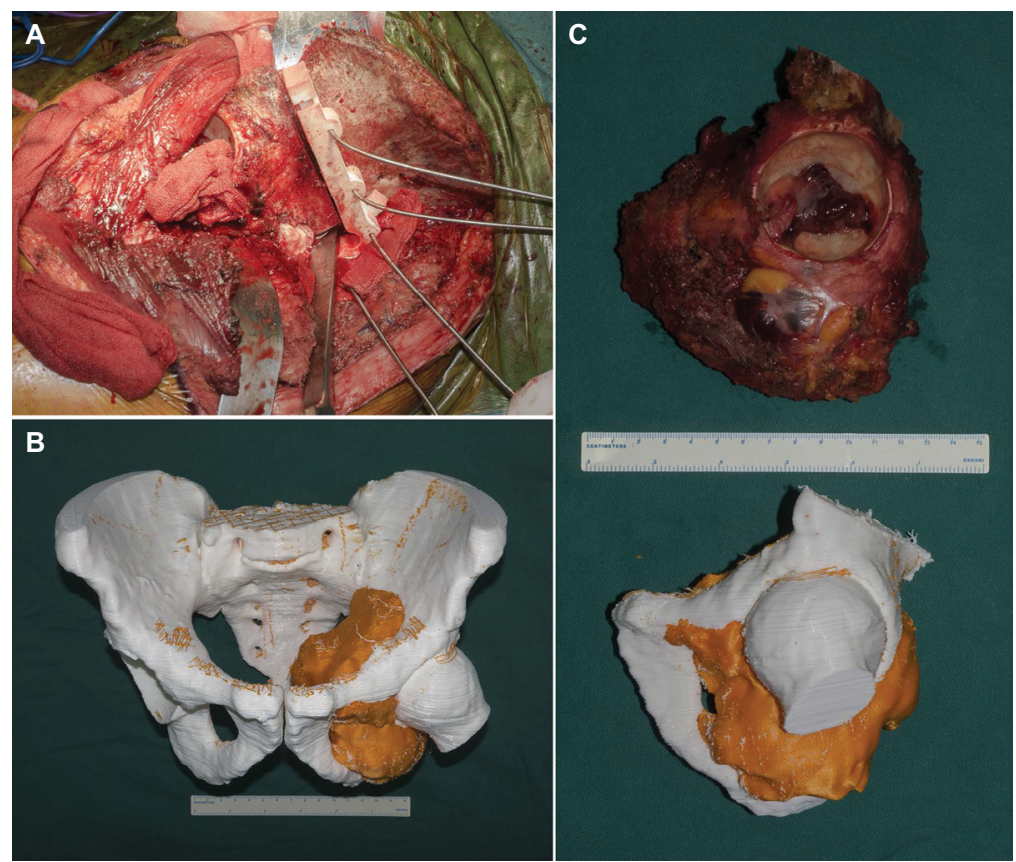

Figure 2 3DMMI-based 3D-printed PSI helped achieve precise resection.

Notes: (A) Intraoperative photo showing osteotomy with PSI. (B) A 3D-printed pelvic model, derived from the 3D-multimodality images, providing intraoperative visual and haptic perception of the surgery. (C) The resected specimen was in high conformity with the simulated surgery, both with 3D-multimodality image-based PSI.

Abbreviations: 3DMMI, 3D-multimodality images; PSI, patient-specific instruments;

Figure 3 3D-multimodality image-based virtual surgical planning and 3D-printed PSI helped the reconstruction of the pelvis after tumor resection.

Notes: (A) An intraoperative photo showing PSI-guided preparation of FH graft, which was used later to fill the gaps between the prosthesis and the ilium. The PSI-I also facilitated the screw fixation by predrilling holes in the $\mathrm{FH}$ graft, combined with PSI-2 in (B). (B) 3D-multimodality image-based virtual surgical planning showing the reconstruction with PSI-2 and the worked $\mathrm{FH}$. PSI-2 was also used to indicate the simulated proper rotation center of the $\mathrm{FH}$. The picture in the left bottom illustrating the gap, between the prosthesis and the ilium, was filled by the FH graft suitably. (C-E) Intraoperative photos and postoperative plain film showing satisfactory reproduction of the virtual surgical planning.

Abbreviations: A, artery; Ba, bladder; FH, femoral head; N, nerve; Pro, prosthesis; Pr, prostate; PSI, patient-specific instruments; Ur, ureter; V, vein. 
tumor and the margin achieved. Prosthesis for reconstruction was provided by ChunLi Co. (Beijing, People's Republic of China). Limited weight-bearing was allowed for $\sim 2$ months for postoperative patients with reconstruction performed. And in patients without reconstruction, immediately weightbearing was allowed.

Surgical margins, complications, duration of surgery, and intra-operative blood loss were analyzed. Data were expressed as median, maximum, and minimum range due to the small sample size. Continuous variables between groups were compared by the Mann-Whitney U test or independentsample $t$-test, according to the Shapiro-Wilk test. Differences in proportions were assessed by Fisher's exact test. Statistical analysis was performed with SPSS Statistics software version 24.0 (IBM Corporation, Armonk, NY, USA); a $P$-value of $<0.05$ was considered statistically significant.

This study was conducted in accordance with the Declaration of Helsinki and with approval from the Ethics Committee of West China Hospital (Chengdu, People's Republic of China). Written informed consent was obtained from all participants.

\section{Results}

In the non-3DMMI group, the margin was wide in six patients $(6 / 13)$, marginal in $4(4 / 13)$, wide-contaminated in $2(2 / 13)$, and intralesional in one (1/13). In this latter patient, intralesional palliative resection was performed due to unexpected intraoperative finding of tumor involvement of first sacral nerve root, fifth lumbar nerve root and sacral vessels. The patient died of pulmonary embolism 1 month postoperatively. And both wide-contaminated margins were actually wide in bone, but intralesional in soft tissue. In the 3DMMI group, all patients underwent surgery as planned. The margin was wide in 10 patients $(10 / 13)$ and marginal in three (3/13). No patients had wide-contaminated or intralesional margins.

The overall mean intra-operative blood loss was 3,450 $\mathrm{mL}(1,000-12,000)$, and this was significantly lower in 3DMMI group $(3,000 \mathrm{~mL} ; 1,000-4,800)$, compared with the non-3DMMI group $(4,200 \mathrm{~mL}, 1,200-12,000 ; P=0.044)$. The mean duration of surgery was shorter in 3DMMI group (393 \pm 92.2 minutes) when compared with the non-3DMMI group $(433 \pm 118.0 ; P=0.354)$.

Three intraoperative complications were seen in the non3DMMI group: one iliac vein tear treated with ligation, one bladder injury treated with repair, and one sciatic nerve injury. In the 3DMMI group, there was only one intraoperative complication. It was difficult to find the unique position for the PSI in one patient, and more soft tissue and periosteum were dissected before the final match.

Postoperative complications occurred in nine patients (9/13) in the non-3DMMI group. Three patients experienced superficial infection treated with dressing. One patient exhibited deep infection treated by debridement, and he also suffered from dislocation 1 week postoperatively treated with open reduction. One patient with intralesional palliative resection had fatal pulmonary embolism and died 1 month after surgery. One patient with sciatic nerve injury presented with foot drop, which resolved 3 months later. Three patients experienced reduced perineal sensation, two of resolved 2 months postoperatively and one still suffered from that in the 6-month follow-up. In the latter case, he also exhibited chronic constipation. In the 3DMMI group, seven patients suffered from postoperative complications. Four patients experienced superficial infection treated with dressing and two patient exhibited deep infection treated by debridement. And one patient exhibited reduced perineal sensation, which resolved 3 months postoperatively. The results are summarized in Table 2 .

\section{Discussion}

Malignant pelvic bone tumors are among the most challenging problems in orthopedic oncology. ${ }^{9}$ It's difficult to achieve wide marginal resection due the complex anatomy of the pelvis, potentially rendering a high risk of local recurrence and vital structures injury. In the last decade, several studies have demonstrated the effectiveness of computer-assisted surgery (CAS), including navigation-assisted surgery and PSI-assisted surgery, in resection and reconstruction of pelvis malignancies. ${ }^{10-14}$ However, most of these reports were designed based on a single or two modalities of image with only a few anatomical structures reconstructed into 3D images. In our study, 3DMMI taking advantages of CT, contrast-enhanced CTA, contrast-enhanced MRI, and contrast-enhanced MRN and revealing the whole tumor and all adjacent critical structures was utilized, for the first time, to aid the virtual surgical planning and 3D-printed PSI for malignancy of the pelvis.

Preoperative surgical evaluation of the extent of malignancy is the basis for limb salvage surgery. Marginal and intralesional resection may result in 70\%-100\% local recurrence. ${ }^{15,16}$ Consequently, accurate preoperative imaging assessment is vital for bone tumors. ${ }^{17,18} \mathrm{CT}$ and MRI are the most effective modalities for this purpose. CT is superior to MRI in detection and characterization of 
Table 2 Intraoperative issues and early outcome

\begin{tabular}{|c|c|c|c|}
\hline & Non-3DMMI group & 3DMMI group & $P$-value \\
\hline Surgical margin & & & 0.275 \\
\hline Wide & 6 & 10 & \\
\hline Marginal & 4 & 3 & \\
\hline Wide-contaminated & 2 & 0 & \\
\hline Intralesional & 1 & 0 & \\
\hline \multicolumn{4}{|l|}{ Complications } \\
\hline Intraoperative & 3 & 1 & 0.593 \\
\hline Vascular injury & 1 & 0 & \\
\hline Nerves injury & 1 & 0 & \\
\hline Bladder injury & 1 & 0 & \\
\hline Poor position of PSI & 0 & I & \\
\hline Postoperative & $9^{a}$ & 7 & \\
\hline Wound infection & 4 & 6 & 0.688 \\
\hline Dislocation & 1 & 0 & \\
\hline Pulmonary embolism & 1 & 0 & \\
\hline Nerves injury & $4^{\mathrm{a}}$ & I & 0.322 \\
\hline Foot-drop & 1 & 0 & \\
\hline Reduced perineal sensation & 3 & I & \\
\hline Bowel dysfunction & I & 0 & \\
\hline Duration of surgery (minutes) & $433 \pm 118.0$ & $393 \pm 92.2$ & 0.354 \\
\hline Blood loss $(\mathrm{mL})^{\mathrm{b}}$ & $4,200(1200,12,000)$ & $3,000(1,000,4800)$ & 0.044 \\
\hline
\end{tabular}

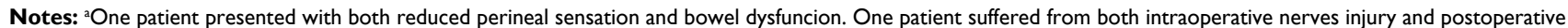
foot-drop. One patient experienced both wound infection and dislocation. ${ }^{\circ}$ Blood loss was expressed as median and range, due to the skewed distribution.

cortical involvement, which enables accurate evaluation of osseous involvement of bone neoplasms. ${ }^{19}$ And intravenous contrast administration is usually indicated to assess the relationship between the neoplasm and vascular bundles. ${ }^{19}$ Due to the high resolution of soft tissue, MRI is superior to CT in evaluation of intramedullary, extraosseous components, and muscle involvement. ${ }^{20} \mathrm{MRN}$ refers to MRI dedicated to peripheral nerves. It is a new technique that depicts neuromuscular imaging alterations directly, which is of high value for surgeons to visualize the consequences of neuromuscular pathology. ${ }^{21,22}$ However, conventional virtual surgical planning mostly depends on a single modality of image, and only a few structures are reconstructed into 3D images. Surgeons still need to build the tumor and its adjacent structures subjectively with each separate image, which may result in inaccurate resection and reconstruction. Abraham et al performed 24 navigation-aided resections of primary pelvic or sacral tumors. ${ }^{10}$ Despite the negative bone margin in all cases, two had positive margin of the soft tissue. Another study also demonstrated 31 negative bone margins with two positive soft tissue margins in 31 cases. ${ }^{23}$ They both performed navigation-assisted surgery based on plain CT/ MRI fusion or a single modality of image. Similarly, in our study, three (3/13) patients in non-3DMMI group had widecontaminated or intralesional margins owing to the massive involvement of the soft tissue mass originated from the bone tumor, compared with full negative margin in all patients from 3DMMI group. Different from simple fusion of two modalities of images conducted with Mimics or navigation system, we invented our own automatic registration and segmentation system based on symmetric diffeomorphic algorithm, $\mathrm{nD}$ morphological contour interpolation, growcut algorithm, region-adaptive registration and Gaussian smoothing, which could make full use of up to four different modalities of images. ${ }^{24-28}$ Nearly all structures, including the congenital anatomy variation and tumor-related variation in the pelvis, could be obtained with true anatomical position in $3 \mathrm{D}$ or $2 \mathrm{D}$ level. With this 3DMMI, preoperative surgical evaluation and planning could be done easily even by less experienced surgeons. Moreover, our virtual surgical plans based on 3DMMI could even be viewed in cellphones (iOS or Android) instead of the computer. Surgical participants could study the anatomy of the specific patients carefully whenever and wherever they are, which might improve intraoperative collaboration and surgical outcomes. Positive margins might thus be avoided.

Due to the proximity to major neurovascular structures and visceral organs, the surgical complication of pelvic malignancy is by no means trivial. In the study by Abraham et al, ${ }^{10}$ two $(2 / 24)$ patients suffered from iliac vein laceration 
during surgery, despite the use of navigation system. Holzapfel et $\mathrm{al}^{29}$ also reported $12.5 \%$ nerves palsy after periacetabular tumors surgery. ${ }^{29}$ In our study, six (6/13) patients in the non-3DMMI group experienced unexpected intraoperative critical structures injury or had presentation of nerves injury postoperatively. The higher rate of injury may result from different inclusion criteria. Only patients with $>10 \mathrm{~cm}$ tumor and large soft tissue mass, but no PSI or navigation was included. And we also recorded reduced perineal sensation (in three patients) as a nerve injury, which was excluded in other studies. However, in the 3DMMI group, there was only one (1/13) such nerve injury. The relatively satisfactory outcome may associate with the precise preoperative surgical evaluation and intraoperative review of 3DMMI, as well as the PSI to reproduce the surgical planning. We noted a higher rate of wound complication in 3DMMI group (4/13 in non3DMMI group, 6/13 in 3DMMI, $P=0.688$ ). However, other studies also reported similar rates on tumors of the pelvis and sacrum, which demonstrated that 3DMMI-based PSI were irrelevant to the wound problems. ${ }^{9,23,30,31}$

Replicating the preoperative plan accurately is also significant in pelvic tumor surgery, in order to achieve safe surgical margins with less unexpected injuries. In 2004, Hüfner et al ${ }^{32}$ first reported navigation-assisted surgery for improved bone resection in pelvic tumors. It has been shown that navigationassisted technique could help decrease intralesional resection rate from $29.0 \%$ to $8.7 \%$, compared with traditional techniques. ${ }^{23}$ Nevertheless, pre-operative and intra-operative errors might occur with navigation-assisted surgery, especially in surface registration. Image-to-patient registration must be checked carefully before resection, ${ }^{33}$ which could be avoided when PSI is utilized, although PSI does require careful preparation of bone surface. Wong et $\mathrm{al}^{34}$ conducted an experimental study comparing navigation- and PSI-assisted surgery in periacetabular tumors. While both groups achieved clinically acceptable accuracy (mean deviation $<2 \mathrm{~mm}$ from planned parameters), PSI is associated with significantly shorter time taken for resection and lower costs than navigation surgery. In this study, the cost for design and manufacture of 3D-printed PSI was $\sim 2$ days and 800 RMB (119 USD), which was costefficient compared with the navigation instruments, similar to the study by Ma et al. ${ }^{35}$ And this technique also helped achieve shorter duration of surgery $(P=0.354)$ and lower intraoperative blood loss $(P=0.044)$ than the non-3DMMI group. However, we did encounter a poor position of PSI issue in one patient, leading to a longer time for the match and resection. Incorrect placement of PSI is fatal, possibly resulting in intralesional resection, injury of vital structures, and poor reconstruction condition of bone stock. PSI would fit better, and there may be less errors for more contoured bone surfaces. ${ }^{36}$ When placed in less contoured surfaces, PSI must be designed much bigger, which requires more intraoperative exposure and dissection. Nevertheless, the surgical exposure is usually extensive for pelvic tumors, which would be adequate for the better fit of the PSI and bone surfaces.

This study had several limitations. First, it was a retrospective non-randomized study. The number of patients was small and the statistical analysis was very limited. Second, it incorporated heterogeneous group of patients with different types of surgery and histological diagnosis, which may limit the strength of conclusions. However, the primary goal for this study was to assess the likelihood of achieving negative surgical margins with less complications utilizing 3DMMIbased techniques, which we felt could be sufficiently studied even in a heterogeneous population. Third, we had a short follow-up in this study. Nevertheless, the focus of this study was not to evaluate the metastases or survival, which would require much longer follow-up and may not be associated with 3DMMI-based CAS; we aimed to evaluate the surgical margins, intraoperative, and postoperative complications, so we believe our follow-up was sufficient for our purpose. Fourth, due to the innate feature of 3DMMI, those invisible in the original images would not be displayed in 3DMMI either. Fifth, limited to the current technical level of CAS, we could not achieve accurate resection of the soft tissue directly, although intraoperative review of the 3DMMI may provide a help in an acceptable resection. Nevertheless, we at least made one step further toward this goal by visualizing all structures, including the congenital anatomy variation and tumor-related variation in the pelvis. Sixth, this casecontrol study only proved that the 3DMMI-based virtual surgical planning and 3DMMI-based PSI may be superior to the traditional surgical plan and technique without CAS. However, the encouraging preliminary results endorse more studies on virtual surgical planning with 3DMMI, based on which precise resection and reconstruction with CAS might be achieved, within, or even beyond the pelvis. ${ }^{37,38}$

\section{Conclusion}

We developed a novel virtual surgical planning system for pelvic tumor surgery by assimilating each separate radiographic image into a single 3DMMI revealing all structures in the pelvis. The 3DMMI provided the basic image data for CAS, which may help achieve negative surgical margin and decrease surgical complications related to critical structures injury for malignant giant pelvic tumor. 


\section{Ethics and copyrights}

Written informed consent has been provided by the patients before this study. And they gave us permission for the materials to appear in the print and online and grant permission to third parties to reproduce these materials.

\section{Acknowledgments}

This work was funded by the Health and Family Planning Commission research project of Sichuan Province (No. 18PJ465), Chengdu Science and Technology Project (2017-CY02-00032-GX), the National Key Research and Development Program of China (No. 2016YFC1102003) and the Science and Technology Research Program of Sichuan Province (No. 2017SZ0095). No benefits in any form have been or will be received from a commercial party related directly or indirectly to the subject of this manuscript. And special thanks to Jianqing Qiu from Department of Epidemiology and Health Statistics, West China School of Public Health, Sichuan University, People's Republic of China, for the statistical assistance regarding this study.

\section{Disclosure}

The authors report no conflicts of interest in this work.

\section{References}

1. Steel HH. Partial or complete resection of the hemipelvis. An alternative to hindquarter amputation for periacetabular chondrosarcoma of the pelvis. J Bone Joint Surg Am. 1978;60(6):719-730.

2. Réguerre Y, Martelli H, Rey A, et al. Local therapy is critical in localised pelvic Rhabdomyosarcoma: experience of the International Society of Pediatric Oncology Malignant Mesenchymal Tumor (SIOP-MMT) committee. Eur J Cancer. 2012;48(13):2020-2027.

3. Delloye C, Banse X, Brichard B, Docquier PL, Cornu O. Pelvic reconstruction with a structural pelvic allograft after resection of a malignant bone tumor. J Bone Joint Surg Am. 2007;89(3):579-587.

4. Mankin HJ, Hornicek FJ, Temple HT, Gebhardt MC. Malignant tumors of the pelvis: an outcome study. Clin Orthop Relat Res. 2004;425(425):212-217.

5. Zhang Y, Guo W, Tang X, et al. En bloc resection of pelvic sarcomas with sacral invasion. Bone Joint J. 2018;100-B(6):798-805.

6. Nowell M, Rodionov R, Zombori G, et al. Utility of 3D multimodality imaging in the implantation of intracranial electrodes in epilepsy. Epilepsia. 2015;56(3):403-413.

7. Enneking WF, Dunham WK. Resection and reconstruction for primary neoplasms involving the innominate bone. J Bone Joint Surg Am. 1978;60(6):731-746.

8. Yushkevich PA, Piven J, Hazlett HC, et al. User-guided 3D active contour segmentation of anatomical structures: significantly improved efficiency and reliability. Neuroimage. 2006;31(3):1116-1128.

9. Bus MPA, Campanacci DA, Albergo JI, et al. Conventional primary central chondrosarcoma of the pelvis: prognostic factors and outcome of surgical treatment in 162 patients. J Bone Joint Surg Am. 2018;100(4):316-325.

10. Abraham JA, Kenneally B, Amer K, Geller DS. Can navigation-assisted surgery help achieve negative margins in resection of pelvic and sacral tumors? Clin Orthop Relat Res. 2018;476(3):499-508.
11. Young PS, Bell SW, Mahendra A. The evolving role of computer-assisted navigation in musculoskeletal oncology. Bone Joint J. 2015;97-B(2): 258-264.

12. Gouin F, Paul L, Odri GA, Cartiaux O. Computer-assisted planning and patient-specific instruments for bone tumor resection within the pelvis: a series of 11 patients. Sarcoma. 2014;2014(270): 842709-9.

13. Jentzsch T, Vlachopoulos L, Fürnstahl P, Müller DA, Fuchs B. Tumor resection at the pelvis using three-dimensional planning and patientspecific instruments: a case series. World J Surg Oncol. 2016;14(1):249.

14. Laitinen MK, Parry MC, Albergo JI, Grimer RJ, Jeys LM. Is computer navigation when used in the surgery of iliosacral pelvic bone tumours safer for the patient? Bone Joint J. 2017;99-B(2):261-266.

15. Ozaki T, Flege S, Kevric M, et al. Osteosarcoma of the pelvis: experience of the Cooperative Osteosarcoma Study Group. J Clin Oncol. 2003;21(2):334-341.

16. Fuchs B, Hoekzema N, Larson DR, Inwards CY, Sim FH. Osteosarcoma of the pelvis: outcome analysis of surgical treatment. Clin Orthop Relat Res. 2009;467(2):510-518.

17. Simon MA, Aschliman MA, Thomas N, Mankin HJ. Limb-salvage treatment versus amputation for osteosarcoma of the distal end of the femur. J Bone Joint Surg Am. 1986;68(9):1331-1337.

18. Lam YL, Yau R, Ho KW, Mak KL, Fong ST, So TY. Is it possible and safe to perform acetabular-preserving resections for malignant neoplasms of the periacetabular region? Clin Orthop Relat Res. 2017;475(3):656-665.

19. Nomikos GC, Murphey MD, Kransdorf MJ, Bancroft LW, Peterson JJ. Primary bone tumors of the lower extremities. Radiol Clin North Am. 2002;40(5):971-990.

20. Greenspan A, Jundt G, Remagen W. Differential Diagnosis in Orthopaedic Oncology. 2nd ed. Philadelphia, PA, USA: Lippincott Williams \& Wilkins; 2007.

21. Thawait SK, Wang K, Subhawong TK, et al. Peripheral nerve surgery: the role of high-resolution MR neurography. AJNR Am J Neuroradiol. 2012;33(2):203-210.

22. Baumer P, Kele H, Kretschmer T, et al. Thoracic outlet syndrome in $3 \mathrm{~T}$ MR neurography-fibrous bands causing discernible lesions of the lower brachial plexus. Eur Radiol. 2014;24(3):756-761.

23. Jeys L, Matharu GS, Nandra RS, Grimer RJ. Can computer navigationassisted surgery reduce the risk of an intralesional margin and reduce the rate of local recurrence in patients with a tumour of the pelvis or sacrum? Bone Joint J. 2013;95-B(10):1417-1424.

24. Cao X, Yang J, Gao Y, Wang Q, Shen D. Region-adaptive deformable registration of CT/MRI pelvic images via learning-based image synthesis. IEEE Trans Image Process. 2018;99:3500-3512.

25. Liangjia Zhu IK, Gao Y, Kikinis R, Tannenbaum A. An effective interactive medical image segmentation method using fast GrowCut. International Conference on Medical Image Computing and Computer Assisted Intervention (MICCAI); 2014; Boston, MA, USA.

26. Avants BB, Epstein CL, Grossman M, Gee JC. Symmetric diffeomorphic image registration with cross-correlation: evaluating automated labeling of elderly and neurodegenerative brain. Med Image Anal. 2008;12(1):26-41.

27. Klein A, Andersson J, Ardekani BA, et al. Evaluation of 14 nonlinear deformation algorithms applied to human brain MRI registration. Neuroimage. 2009;46(3):786-802.

28. Murphy K, van Ginneken B, Reinhardt JM, et al. Evaluation of registration methods on thoracic CT: the EMPIRE10 challenge. IEEE Trans Med Imaging. 2011;30(11):1901-1920.

29. Holzapfel BM, Pilge H, Prodinger PM, et al. Customised osteotomy guides and endoprosthetic reconstruction for periacetabular tumours. Int Orthop. 2014;38(7):1435-1442.

30. Bus MP, Szafranski A, Sellevold S, et al. LUMiC ${ }^{\circledR}$ endoprosthetic reconstruction after periacetabular tumor resection: short-term results. Clin Orthop Relat Res. 2017;475(3):686-695.

31. Cho HS, Oh JH, Han I, Kim HS. The outcomes of navigation-assisted bone tumour surgery: minimum three-year follow-up. J Bone Joint Surg Br. 2012;94(10):1414-1420. 
32. Hüfner T, Kfuri M, Galanski M, et al. New indications for computerassisted surgery: tumor resection in the pelvis. Clin Orthop Relat Res. 2004;(426):219-225.

33. Morishige M, Mcqueen DA, Chong A, Ballard GP, Cooke FW. The influence of sequential debridement in total knee arthroplasty on the flexion axis of the knee using computer-aided navigation. J Orthop Res. 2010;28(6):767-772.

34. Wong KC, Sze KY, Wong IO, Wong CM, Kumta SM. Patient-specific instrument can achieve same accuracy with less resection time than navigation assistance in periacetabular pelvic tumor surgery: a cadaveric study. Int J Comput Assist Radiol Surg. 2016;11(2):307-316.
35. Ma L, Zhou Y, Zhu Y, et al. 3D-printed guiding templates for improved osteosarcoma resection. Sci Rep. 2016;6:23335.

36. Wong KC. 3D-printed patient-specific applications in orthopedics. Orthop Res Rev. 2016;8(8):57-66.

37. Fang X, Liu H, Xiong Y, et al. Total talar replacement with a novel 3D printed modular prosthesis for tumors. Ther Clin Risk Manag. 2018;14: 1897-1905.

38. Fang X, Zhang W, Yu Z, et al. Total ulna replacement with a 3D-printed custom-made prosthesis after en bloc tumor resection: a case report. Pediatr Blood Cancer. 2018:e27522.
Cancer Management and Research

\section{Publish your work in this journal}

Cancer Management and Research is an international, peer-reviewed open access journal focusing on cancer research and the optimal use of preventative and integrated treatment interventions to achieve improved outcomes, enhanced survival and quality of life for the cancer patient The manuscript management system is completely online and includes a very quick and fair peer-review system, which is all easy to use. Visit http://www.dovepress.com/testimonials.php to read real quotes from published authors. 